\title{
Flavour violating up-squark decays at LHC
}

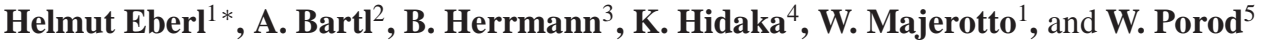 \\ ${ }^{1}$ Institut für Hochenergiephysik der ÖAW, Nikolsdorfer Gasse 18, 1050 Wien, Austria \\ ${ }^{2}$ Fakultät für Physik, Universität Wien, A-1090 Vienna, Austria \\ ${ }^{3}$ Deutsches Elektronen-Synchrotron (DESY), Theory Group, D-22603 Hamburg, Germany \\ ${ }^{4}$ Department of Physics, Tokyo Gakugei University, Koganei, Tokyo 184-8501, Japan \\ ${ }^{5}$ Institut für Theoretische Physik und Astrophysik, Universität Würzburg, D-97074 Würzburg, Germany
}

Key words Supersymmetry, MSSM, squarks, flavour violation, LHC

Subject classification $11.30 . \mathrm{Pb}, 11.30 . \mathrm{Hv}, 12.60 . \mathrm{Jv}, 13.85$.-t, $14.80 . \mathrm{Ly}$

\begin{abstract}
We study the effect of squark generation mixing on squark production and decays at the LHC in the Minimal Supersymmetric Standard Model (MSSM). We show that the effect can be very large despite the very strong constraints on quark flavour violation (QFV) from experimental data on B mesons. We find that the two lightest up-type squarks $\tilde{u}_{1,2}$ can have large branching ratios for the decays into $c \tilde{\chi}_{1}^{0}$ and $t \tilde{\chi}_{1}^{0}$ at the same time, leading to QFV signals ' $p p \rightarrow c \bar{t}(t \bar{c})+$ missing- $E_{T}+X$ ' with a significant rate. The observation of this remarkable signature would provide a powerful test of supersymmetric QFV at LHC. This could have a significant impact on the search for squarks and the determination of the underlying MSSM parameters.
\end{abstract}

Copyright line will be provided by the publisher

\section{Introduction}

The Minimal Supersymmetric Standard Model is the most intensively studied extension of the Standard Model (SM) of elementary particles. Low energy Supersymmetry (SUSY) provides a solution to the socalled hierarchy problem in the Higgs sector, allows gauge coupling unification, and provides possible candidates for the dark matter observed in our Universe. If the MSSM is realized in nature, LHC will copiously produce gluinos and squarks, the supersymmetric partners of gluons and quarks, for masses of $O(1 \mathrm{TeV})$. However, even if SUSY is discovered, it will be still a long way to determine the parameters of the underlying model, which would shed light on the mechanism of SUSY breaking. So-called softSUSY-breaking terms are introduced in the SUSY Lagrangian. As these soft-SUSY-breaking terms could also be the source of flavour violation beyond the Standard Model (SM), the measurement of flavour violating observables is directly linked to the crucial question about the SUSY-breaking mechanism. It is usually assumed that production and decays of squarks are quark-flavour conserving (QFC). However, additional flavour structures (i.e. squark generation mixings) would imply that squarks are not quarkflavour eigenstates, which could result in sizable quark-flavour violation (QFV) effects significantly larger than those due to the Cabibbo-Kobayashi-Maskawa(CKM) mixing. In this contribution we study the effect of QFV due to the mixing of charm-squarks and top-squarks on production and decays of squarks at the LHC in the general MSSM.

\section{Squark mixing with flavour violation and constraints}

The most general up-type squark mass matrix including left-right mixing as well as quark-flavour mixing in the super-CKM basis of $\tilde{u}_{0 \gamma}=\left(\tilde{u}_{L}, \tilde{c}_{L}, \tilde{t}_{L}, \tilde{u}_{R}, \tilde{c}_{R}, \tilde{t}_{R}\right), \gamma=1, \ldots, 6$, is [1]

\footnotetext{
* Speaker, e-mail: helmut@hephy.oeaw.ac.at, Phone: +43(0)1 544732835 , Fax: +43 (0)1 544732854
} 


$$
\begin{aligned}
& M_{\tilde{u}}^{2}=\left(\begin{array}{cc}
M_{\tilde{u} L L}^{2} & \left(M_{\tilde{u} R L}^{2}\right)^{\dagger} \\
M_{\tilde{u} R L}^{2} & M_{\tilde{u} R R}^{2}
\end{array}\right), \quad \text { where the three } 3 \times 3 \text { matrices read } \\
& \left(M_{\tilde{u} L L}^{2}\right)_{\alpha \beta}=M_{Q_{u} \alpha \beta}^{2}+\left[\left(\frac{1}{2}-\frac{2}{3} \sin ^{2} \theta_{W}\right) \cos 2 \beta m_{Z}^{2}+m_{u_{\alpha}}^{2}\right] \delta_{\alpha \beta}, \\
& \left(M_{\tilde{u} R R}^{2}\right)_{\alpha \beta}=M_{U \alpha \beta}^{2}+\left[\frac{2}{3} \sin ^{2} \theta_{W} \cos 2 \beta m_{Z}^{2}+m_{u_{\alpha}}^{2}\right] \delta_{\alpha \beta}, \\
& \left(M_{\tilde{u} R L}^{2}\right)_{\alpha \beta}=\left(v_{2} / \sqrt{2}\right) T_{U \beta \alpha}-m_{u_{\alpha}} \mu^{*} \cot \beta \delta_{\alpha \beta} .
\end{aligned}
$$

The indices $\alpha, \beta=1,2,3$ characterize the quark flavours $u, c, t$, respectively. $M_{Q_{u}}^{2}$ and $M_{U}^{2}$ are the hermitean soft-SUSY-breaking mass matrices for the left and right up-type squarks, respectively. $T_{U}$ is the soft-SUSY-breaking trilinear coupling matrix of the up-type squarks. The physical mass eigenstates $\tilde{u}_{i}$, $i=1, \ldots, 6$, are given by $\tilde{u}_{i}=R_{i \alpha}^{\tilde{u}} \tilde{u}_{0 \alpha}$. The mixing matrix $R^{\tilde{u}}$ and the mass eigenvalues are obtained by a unitary transformation $R^{\tilde{u}} M_{\tilde{u}}^{2} R^{\tilde{u} \dagger}=\operatorname{diag}\left(m_{\tilde{u}_{1}}^{2}, \ldots, m_{\tilde{u}_{6}}^{2}\right)$, where $m_{\tilde{u}_{i}}<m_{\tilde{u}_{j}}$ for $i<j$.

We define dimensionless QFV parameters $\delta_{\alpha \beta}^{u L L}$ and $\delta_{\alpha \beta}^{u R R}$ as

$$
\delta_{\alpha \beta}^{u L L} \equiv M_{Q \alpha \beta}^{2} / \sqrt{M_{Q \alpha \alpha}^{2} M_{Q \beta \beta}^{2}}, \quad \delta_{\alpha \beta}^{u R R} \equiv M_{U \alpha \beta}^{2} / \sqrt{M_{U \alpha \alpha}^{2} M_{U \beta \beta}^{2}},
$$

for $\delta_{\alpha \beta}^{u R L}$ see [1]. For example, $\delta_{23}^{u L L}$ and $\delta_{23}^{u R R}$ are the $\tilde{c}_{L}-\tilde{t}_{L}$ and $\tilde{c}_{R}-\tilde{t}_{R}$ mixing parameters. In our analysis, we impose the following conditions on the MSSM parameter space in order to respect experimental and theoretical constraints, for details see [1]:

i) Constraints from the B-physics experiments [2], such as $2.92 \times 10^{-4}<B(b \rightarrow s \gamma)<4.22 \times 10^{-4}$, $\left|\Delta M_{B_{s}}^{\mathrm{SUSY}}-17.77\right|<3.31 \mathrm{ps}^{-1}$ and those from other rare B-decays

ii) The experimental limit on the SUSY contributions to the $\rho$ parameter

iii) LEP and Tevatron lower limits on the SUSY particle masses

iv) Vacuum stability conditions for the elements of the trilinear coupling matrices $T_{U}$ and $T_{D}$

The conditions i) and iv) strongly constrain the $2^{\text {nd }}$ and $3^{\text {rd }}$ generation squark mixing parameters $M_{Q 23}^{2}$, $M_{D 23}^{2}, T_{U 23}, T_{D 23}$, and $T_{D 32}$, but not $M_{U 23}^{2}$ which is parametrized by $\delta_{23}^{u R R}$. In the following numerical presentation all of these constraints are fulfilled.

\section{Flavour violating fermionic squark decays at LHC}

First we study the effect of the mixing between the $2^{\text {nd }}$ and $3^{\text {rd }}$ generation of up-squarks on their decays only. The widths of the two-body decays

$$
\tilde{u}_{i} \rightarrow u_{k} \tilde{g}, u_{k} \tilde{\chi}_{n}^{0}, d_{k} \tilde{\chi}_{m}^{+}, \tilde{u}_{j} Z^{0}, \tilde{d}_{j} W^{+}, \tilde{u}_{j} h^{0}
$$

are calculated, where $u_{k}=(u, c, t)$ and $d_{k}=(d, s, b)$. A specific numerical scenario is chosen so that QFV signals at LHC are large. It is given in Table 1 In this scenario one has $\delta_{23}^{u L L}=0.068, \delta_{23}^{u R R}=0.4$ and $\delta_{23}^{u R L}=\delta_{32}^{u R L}=0$ for the QFV parameters. We obtain (masses in $\mathrm{GeV}$ ):

$m_{\tilde{u}_{1}}=472, m_{\tilde{u}_{2}}=708, \tilde{u}_{1} \sim-0.72 \tilde{c}_{R}+0.70 \tilde{t}_{R}, \tilde{u}_{2} \sim 0.70 \tilde{c}_{R}+0.71 \tilde{t}_{R}, m_{\tilde{\chi}_{1}^{0}}=138$, and the branching $\operatorname{ratios} B\left(\tilde{u}_{1} \rightarrow c \tilde{\chi}_{1}^{0}\right)=0.59, B\left(\tilde{u}_{1} \rightarrow t \tilde{\chi}_{1}^{0}\right)=0.39, B\left(\tilde{u}_{2} \rightarrow c \tilde{\chi}_{1}^{0}\right)=0.44, B\left(\tilde{u}_{2} \rightarrow t \tilde{\chi}_{1}^{0}\right)=0.40$. Note that the branching ratios of the decays of a squark into quarks of different generations are very large 


\begin{tabular}{|c||c|c|c|c|c|c|c|c|c|}
\hline$M_{Q \alpha \beta}^{2}$ & $\beta=1$ & $\beta=2$ & $\beta=3$ & \multicolumn{1}{|c|}{} \\
\hline \hline$\alpha=1$ & $(920)^{2}$ & 0 & 0 & $M_{1}$ & $M_{2}$ & $M_{3}$ & $\mu$ & $\tan \beta$ & $m_{A^{0}}$ \\
\hline \hline$\alpha=2$ & 0 & $(880)^{2}$ & $(224)^{2}$ & 139 & 264 & 800 & 1000 & 10 & 800 \\
\hline$\alpha=3$ & 0 & $(224)^{2}$ & $(840)^{2}$ \\
\hline
\end{tabular}

\begin{tabular}{|c||c|c|c|}
\hline$M_{D \alpha \beta}^{2}$ & $\beta=1$ & $\beta=2$ & $\beta=3$ \\
\hline \hline$\alpha=1$ & $(830)^{2}$ & 0 & 0 \\
\hline$\alpha=2$ & 0 & $(820)^{2}$ & 0 \\
\hline$\alpha=3$ & 0 & 0 & $(810)^{2}$ \\
\hline
\end{tabular}

\begin{tabular}{|c||c|c|c|}
\hline$M_{U \alpha \beta}^{2}$ & $\beta=1$ & $\beta=2$ & $\beta=3$ \\
\hline \hline$\alpha=1$ & $(820)^{2}$ & 0 & 0 \\
\hline$\alpha=2$ & 0 & $(600)^{2}$ & $(373)^{2}$ \\
\hline$\alpha=3$ & 0 & $(373)^{2}$ & $(580)^{2}$ \\
\hline
\end{tabular}

Table 1 Input parameters for the reference scenario (mass parameters in $\mathrm{GeV}$ ). All $T_{U \alpha \beta}$ and $T_{D \alpha \beta}$ are zero.
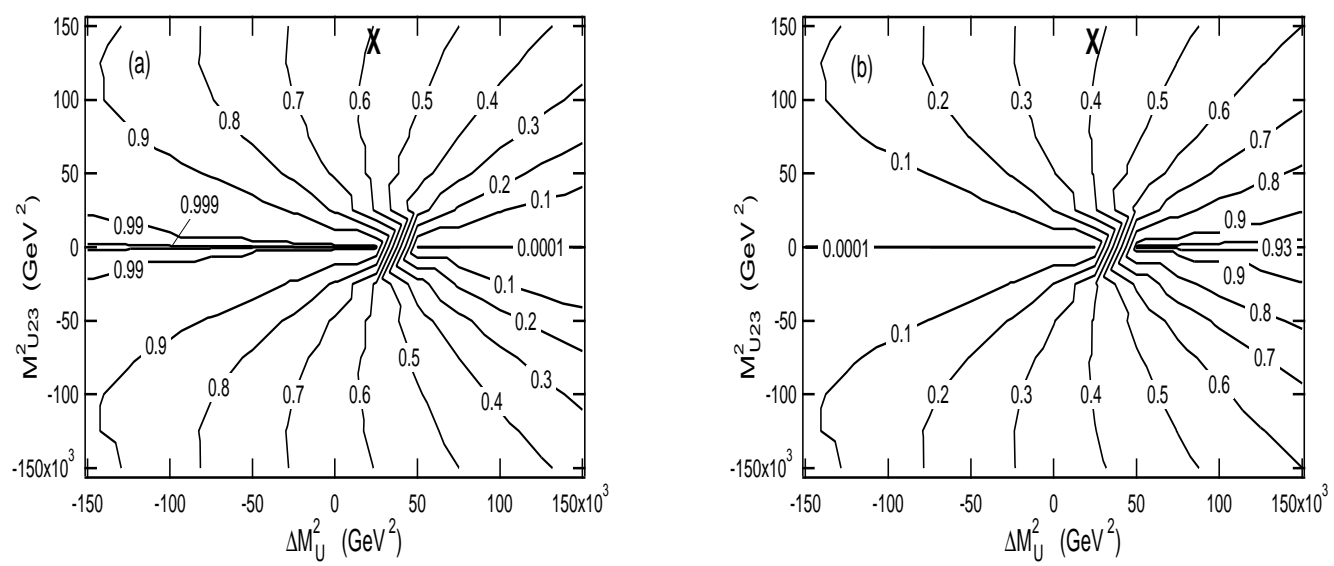

Fig. 1 Contours of QFV decay branching ratios (a) $B\left(\tilde{u}_{1} \rightarrow c \tilde{\chi}_{1}^{0}\right)$ and (b) $B\left(\tilde{u}_{1} \rightarrow t \tilde{\chi}_{1}^{0}\right)$ in the $\left(\Delta M_{U}^{2}, M_{U 23}^{2}\right)$ plane.

simultaneously, which could lead to large QFV effects. In our scenario this is a consequence of the fact that both squarks $\tilde{u}_{1,2}$ are mainly strong mixtures of $\tilde{c}_{R}$ and $\tilde{t}_{R}$ due to the large $\tilde{c}_{R}-\tilde{t}_{R}$ mixing term $M_{U 23}^{2}\left(=(373 \mathrm{GeV})^{2}\right)$ and that $\tilde{\chi}_{1}^{0}$ is mainly the $U(1)$ gaugino. This also suppresses the couplings of $\tilde{u}_{1,2}$ to $\tilde{\chi}_{2}^{0}$ and $\tilde{\chi}_{1}^{+}$which are mainly $S U(2)$ gauginos. Note that $\tilde{\chi}_{3,4}^{0}$ and $\tilde{\chi}_{2}^{ \pm}$are very heavy in this scenario.

Fig. 11 shows the contours of $B\left(\tilde{u}_{1} \rightarrow c \tilde{\chi}_{1}^{0}\right)$ and $B\left(\tilde{u}_{1} \rightarrow t \tilde{\chi}_{1}^{0}\right)$ in the $\left(\Delta M_{U}^{2}, M_{U 23}^{2}\right)$ plane with $\Delta M_{U}^{2} \equiv$ $M_{U 22}^{2}-M_{U 33}^{2}$. The range of $M_{U 23}^{2}$ shown corresponds to the range $\left|\delta_{23}^{u R R}\right|<0.45$ for $\Delta M_{U}^{2}=0$. In all plots the point corresponding to the scenario given in Table 1 is marked by an " $x$ ". We see that there are sizable regions where both decay modes are important at the same time. The observed behavior can be easily understood in the limit where the $\tilde{t}_{L}-\tilde{t}_{R}$ mixing is neglected since in this limit only the mixing between $\tilde{c}_{R}$ and $\tilde{t}_{R}$ is relevant for $\tilde{u}_{1,2}$ and the corresponding effective mixing angle is given by $\tan \left(2 \theta_{\tilde{c}_{R} \tilde{t}_{R}}^{\text {eff }}\right) \equiv 2 M_{U 23}^{2} /\left(\Delta M_{U}^{2}-m_{t}^{2}\right)$. Note that for $\Delta M_{U}^{2}-m_{t}^{2}>0\left[\Delta M_{U}^{2}-m_{t}^{2}<0\right]$, we have $\tilde{u}_{1} \sim$ $\tilde{t}_{R}\left(+\tilde{c}_{R}\right)\left[\tilde{u}_{1} \sim \tilde{c}_{R}\left(+\tilde{t}_{R}\right)\right]$. We further study effects of the squark generation mixing on QFV signals at LHC. The large $B\left(\tilde{u}_{i} \rightarrow c \tilde{\chi}_{1}^{0}\right)$ and $B\left(\tilde{u}_{i} \rightarrow t \tilde{\chi}_{1}^{0}\right)(i=1,2)$ may result in a sizable rate for the following QFV signals:

$$
p p \rightarrow \tilde{u}_{1,2} \overline{\tilde{u}}_{1,2} X \rightarrow c \bar{t} \tilde{\chi}_{1}^{0} \tilde{\chi}_{1}^{0} X, t \bar{c} \tilde{\chi}_{1}^{0} \tilde{\chi}_{1}^{0} X
$$

where $\mathrm{X}$ contains only beam jets and the $\tilde{\chi}_{1}^{0}$ 's give rise to missing transverse energy $E_{T}^{m i s}$. Defining

$$
\sigma_{c t}^{i j} \equiv \sigma\left(p p \rightarrow \tilde{u}_{i} \overline{\tilde{u}}_{j} X\right) \times\left(B\left(\tilde{u}_{i} \rightarrow c \tilde{\chi}_{1}^{0}\right) \times B\left(\overline{\tilde{u}}_{j} \rightarrow \bar{t} \tilde{\chi}_{1}^{0}\right)+B\left(\tilde{u}_{i} \rightarrow t \tilde{\chi}_{1}^{0}\right) \times B\left(\overline{\tilde{u}}_{j} \rightarrow \bar{c} \tilde{\chi}_{1}^{0}\right)\right)
$$



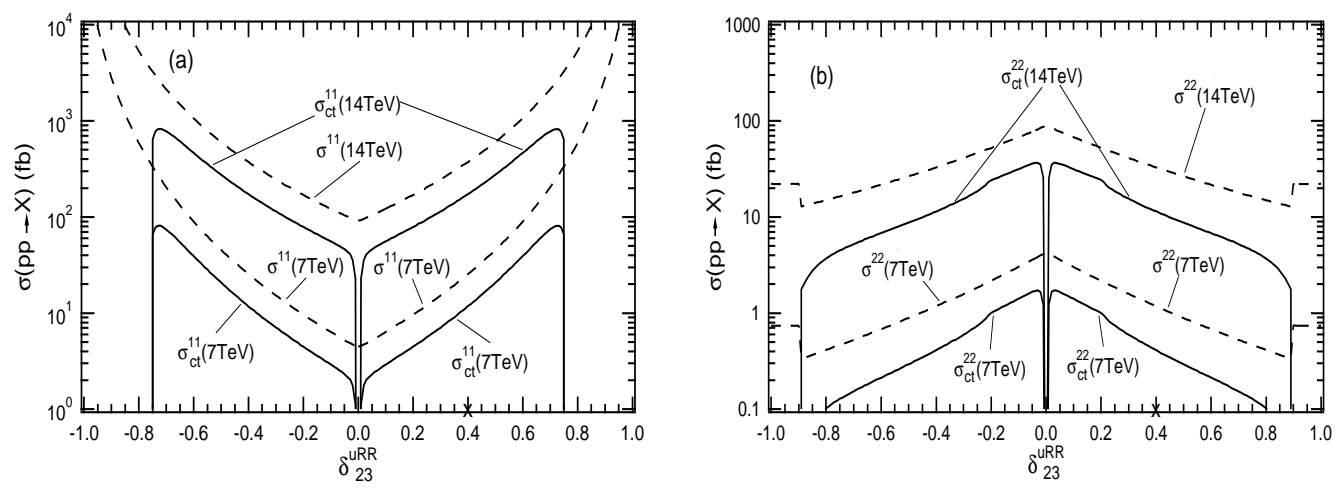

Fig. $2 \delta_{23}^{u R R}$ dependences of (a) $\sigma^{11} \equiv \sigma\left(p p \rightarrow \tilde{u}_{1} \overline{\tilde{u}}_{1} X\right), \sigma_{c t}^{11}$ and (b) $\sigma^{22} \equiv \sigma\left(p p \rightarrow \tilde{u}_{2} \overline{\tilde{u}}_{2} X\right), \sigma_{c t}^{22}$.

we obtain the following cross sections at $E_{c m}=14 \mathrm{TeV}[7 \mathrm{TeV}]$ for the scenario of Table 1 . $\sigma_{c t}^{11}=172.8[11.8] \mathrm{fb}, \sigma_{c t}^{22}=11.5[0.41] \mathrm{fb}$.

In Fig. 2] we show the $\delta_{23}^{u R R}$ dependences of the QFV production cross sections $\sigma_{c t}^{i i}(i=1,2)$ at $E_{c m}$ $=7$ and $14 \mathrm{TeV}$, where all basic parameters other than $M_{U 23}^{2}$ are fixed as in the scenario of Table 1 . The QFV cross sections at $14 \mathrm{TeV}$ are about an order of magnitude larger than those at $7 \mathrm{TeV}$. We see that the QFV cross sections quickly increase with increase of the QFV parameter $\left|\delta_{23}^{u R R}\right|$ around $\delta_{23}^{u R R}=0$ and that they can be quite sizable in a wide allowed range of $\delta_{23}^{u R R}$. The mass of $\tilde{u}_{1}\left(\tilde{u}_{2}\right)$ decreases (increases) with increase of $\left|\delta_{23}^{u R R}\right|$. This leads to the increase of $\sigma_{c t}^{11}$ and the decrease of $\sigma_{c t}^{22}$ with increase of $\left|\delta_{23}^{u R R}\right| . \sigma_{c t}^{11}$ vanishes for $\left|\delta_{23}^{u R R}\right| \gtrsim 0.76$, where the decay $\tilde{u}_{1} \rightarrow t \tilde{\chi}_{1}^{0}$ is kinematically forbidden. We have $\tilde{u}_{2}=\tilde{u}_{R}$ for $\left|\delta_{23}^{u R R}\right| \gtrsim 0.9$, which explains the enhancement of $\sigma\left(p p \rightarrow \tilde{u}_{2} \overline{\tilde{u}}_{2} X\right)$ and the vanishing of $\sigma_{c t}^{22}$ for $\left|\delta_{23}^{u R R}\right| \gtrsim 0.9$. We see that the expected number of signal events at the LHC would be up to about 20000 (10) for an integrated luminosity of $100 \mathrm{fb}^{-1}\left(1 \mathrm{fb}^{-1}\right)$ at $\sqrt{s}=14 \mathrm{TeV}(7 \mathrm{TeV})$. Concerning the detectability, top-quark identification is necessary to distinguish the proposed signal from $t \bar{t}$ production including missing energy. Efficient charm-tagging would be very helpful for detecting flavour mixing between the second and third generation. Otherwise, one should rather search for the signature jet + $t(\bar{t})+E_{T}^{\text {miss }}[1]$.

We remark that for the QFV scenarios based on a mSUGRA scenario such as SPS1a' we have obtained results similar to the presented one [1].

In summary, despite the strong constraints from experimental data, non-minimal QFV can lead to new signatures at LHC. This has been shown explicitly for one scenario. The given conclusions hold, however, for a wide range of the MSSM parameter space [1]. Of course, the next necessary step is a detailed MonteCarlo study including background reactions and detector simulation in order to identify the parameter region where the proposed QFV signal is observable with sufficient significance.

\section{Acknowledgements}

The authors acknowledge support from the "Fonds zur Förderung der wissenschaftlichen Forschung" of Austria, project No. P 18959-N16 and project No. I 297-N16, and from the DFG, project nr. PO-1337/2-1. This work is supported in part by the Landes-Exzellenzinitiative Hamburg.

\section{References}

[1] A. Bartl, H. Eberl, B. Herrmann, K. Hidaka, W. Majerotto, W. Porod, Phys. Lett. B 698 (2011) 380 [arXiv:1007.5483 [hep-ph]]; Erratum to be published.

[2] See corresponding references in [1]. 